


\title{
Fabrication of semi-epitaxial Fe microdots on GaAs (100) substrates
}

Nilay Gunduz Akdogan

Online Publication Date: 27 Feb 2021

URL: http://www.jresm.org/archive/rresm2020.233na1116.html

DOI: http://dx.doi.org/10.17515/resm2020.233na1116

Journal Abbreviation: Res. Eng. Struct. Mater.

\section{To cite this article}

Gunduz Akdogan N. Fabrication of semi-epitaxial Fe microdots on GaAs (100) substrates. Res. Eng. Struct. Mater., 2021; 7(2): 273-279.

\section{Disclaimer}

All the opinions and statements expressed in the papers are on the responsibility of author(s) and are not to be regarded as those of the journal of Research on Engineering Structures and Materials (RESM) organization or related parties. The publishers make no warranty, explicit or implied, or make any representation with respect to the contents of any article will be complete or accurate or up to date. The accuracy of any instructions, equations, or other information should be independently verified. The publisher and related parties shall not be liable for any loss, actions, claims, proceedings, demand or costs or damages whatsoever or howsoever caused arising directly or indirectly in connection with use of the information given in the journal or related means.

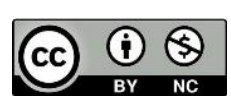

Published articles are freely available to users under the terms of Creative Commons Attribution - NonCommercial 4.0 International Public License, as currently displayed at here (the "CC BY - NC"). 


\title{
Research on Engineering Structures \& Materials
}

journal homepage: http://jresm.org

\section{Fabrication of semi-epitaxial Fe microdots on GaAs (100) substrates}

\author{
Nilay Gunduz Akdogan
}

Faculty of Engineering, Piri Reis University, 34940 Istanbul, Turkey

\begin{tabular}{|c|c|}
\hline Article Info & Abstract \\
\hline $\begin{array}{l}\text { Article history: } \\
\text { Received } 16 \text { Nov } 2020 \\
\text { Revised 05 Feb } 2021 \\
\text { Accepted } 25 \text { Feb } 2021\end{array}$ & $\begin{array}{l}\text { Fe thin films and micro-dots were deposited onto } \mathrm{Si}(001) \text { and GaAs (100) } \\
\text { substrates by a combinatorial approach of lithography and e-beam deposition } \\
\text { techniques. The base pressure in the evaporation chamber was } 1 \times 10^{-6} \text { mbar. } \\
50 \mathrm{~nm} \text { and } 5 \mathrm{~nm} \text { of Fe was deposited with a deposition rate of } 0.1 \mathrm{~nm} / \mathrm{sec} \text { onto } \mathrm{Si} \\
\text { and GaAs, respectively. Continuous films and microdots were covered with } 5 \mathrm{~nm}\end{array}$ \\
\hline $\begin{array}{l}\text { Keywords: } \\
\text { Fe/GaAs; micro-dots; } \\
\text { magnetic anisotropy; } \\
\text { MEMS }\end{array}$ & $\begin{array}{l}\mathrm{Cr} \text { to prevent oxidation. In-plane interfacial uniaxial magnetic anisotropy has } \\
\text { been observed in micro-dots. Room temperature coercivity of } 925 \text { oe has been } \\
\text { observed in out-of-plane direction. Effects of strain and interfacial phases on in- } \\
\text { plane uniaxial anisotropy are discussed. Synthesized micro-dots could be the } \\
\text { building blocks for next-generation magnetoelectronic devices. }\end{array}$ \\
\hline
\end{tabular}

(C) 2021 MIM Research Group. All rights reserved.

\section{Introduction}

Since the first observation of epitaxy in Fe films deposited on GaAs substrate, the system attracted quite a bit of attention from the research community. [1] This is due to the system's suitability to be used in spin-based magneto-electric device applications (spintronic). [2] Among the many hybrid Ferromagnetic/Semiconductor (FM/SC) systems, Fe and GaAs have special status not only due to their low lattice mismatch $(\eta=1.4 \%)[3,4]$ but also due to the high curie temperature of Fe and widely known III-V SC substrate; GaAs. Some other possible SC substrate candidates are $\mathrm{ZnSe}$, InAs, AlAs, Ge, etc.

Even though the spin injection efficiencies are reasonably lower compared to other SC devices [5], it has been shown that interface engineering could improve the spin injection efficiency of the Fe/GaAs system considerably. [6-8]

Several difficulties relating to the $\mathrm{Fe} / \mathrm{GaAs}$ interface are known in the literature. A hightemperature deposition is necessary to increase the surface mobility but also causes the out-diffusion of substrate atoms into the thin film. Ergo, a wide range of deposition temperatures from $-150{ }^{\circ} \mathrm{C}(9]$ to RT $[1,10]$ and even to $580^{\circ} \mathrm{C}[11]$ have been applied. It is widely known that out-diffusion of As (Even $\mathrm{Ga}$ ) into the Fe film is a big problem since Fe atoms prefer bonding to As over Ga. [12] Even segregation of As has been observed on the surface of the Fe films. [13] Besides, it has been observed that the As segregation (surface diffusion) is possible at all temperatures $\left(-15^{\circ} \mathrm{C}\right.$ to $\left.175^{\circ} \mathrm{C}\right)[10,14-16]$

Filipe et al. [17] showed that the out-diffused As atoms form Fe-Ga-As (ferromagnetic, $\mathrm{Hc}: 50 \mathrm{Oe}$, has a fraction of Fe's magnetic moment) and $\mathrm{Fe}_{2} \mathrm{As}$ (antiferromagnetic) at the interface, thus leading to an epitaxial Fe/Reacted-phases/GaAs hybrid structure. Several

Corresponding author: ngakdogan@pirireis.edu.tr orcid.org/0000-0001-7268-0605; DOI: $\underline{\text { http://dx.doi.org/10.17515/resm2020.233na1116 }}$

Res. Eng. Struct. Mat. Vol. 7 Iss. 2 (2021) 273-279 
groups tried to experiment with surface temperature, surface termination, and preventive buffer layers (Ag [18], Al [9], S [19] and Oxide [20]) to prevent the out-diffusion, but either the buffer layer did not prevent the diffusion or caused other problems such as shunting the magnetoelectric device or distorting epitaxy.

The main interest of this work and the subject of ongoing scientific speculations is the observed interplay between four-fold bulk like in-plane magnetic anisotropy and two-fold uniaxial in-plane magnetic anisotropy. While the latter is dominant in ultrathin Fe films, the former is governing the thicker and bulk dimensions. [2] In-plane uniaxial anisotropy is believed to be caused by the interfacial phases and/or magnetoelastic strain. Magnetic anisotropy will be discussed in detail later on.

In this study, $5 \mathrm{~nm}$ thick Fe micro-dots have been successfully deposited onto the GaAs substrate by using the combination of lithography and e-beam evaporation techniques and magnetic properties have been discussed. Such microdots could be used in future magnetoelectric devices and MEMS.

\section{Experimental}

Fe micro-dots were synthesized by the lift-off process. GaAs (100) and Si (001) wafers were used as substrates. Substrates were spin-coated at a speed of $4000 \mathrm{rpm}$ for $45 \mathrm{sec}$. with Az5214e photoresist. The coated resist was baked to harden at $105^{\circ} \mathrm{C}$ for $1 \mathrm{~min}$. Next, substrates were subjected to a UV-light ( $4.5 \mathrm{sec}$.) through the prefabricated mask with a pattern of $100 \mu \mathrm{m}$ diameter circles decorated $100 \mu \mathrm{m}$ apart. (Midas/MDA-60MS Mask Aligner 4') UV lights weaken the links of the exposed parts of the resist coating, which was later removed by 726 MIF developer. The prepared array of circular holes was filled by depositing (Torr E-beam and Thermal Evaporator) $50 \mathrm{~nm}$ Fe (deposition rate: $0.1 \mathrm{~nm} / \mathrm{sec}$ ) ( $5 \mathrm{~nm}$ on GaAs) and $5 \mathrm{~nm} \mathrm{Cr}$ cover layer (to prevent oxidation). The base pressure in the evaporation chamber was $1 \times 10^{-6}$ mbar. (Scheme 1)

\subsection{Characterization:}

Morphology analyses of the samples were performed using Jeol JSM 6010. Magnetic measurements at room temperature were made with a Quantum Design VSM-SQUID. Structural characterizations were performed using Bruker D8 X-ray diffractometer (XRD) using a monochromatic $\mathrm{Cu} \mathrm{K} \alpha \mathrm{X}$-ray source.

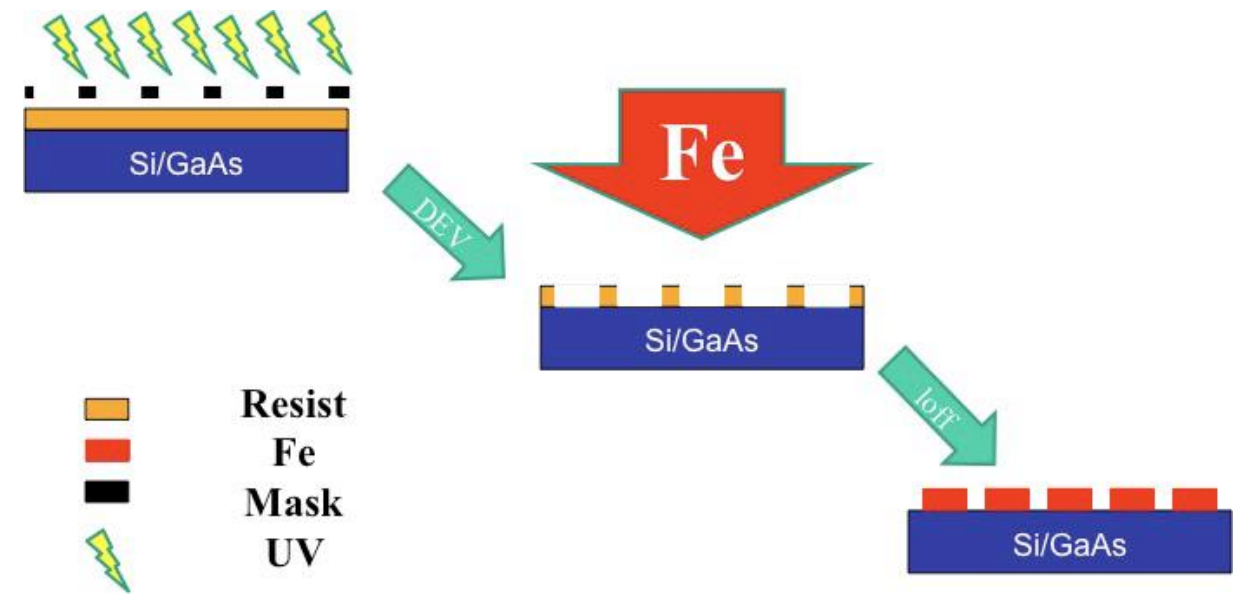

Scheme. 1 Schematic of the experimental process. 


\section{Results and Discussion}

In order to have a base data for the films deposited on GaAs, Fe continuous thin film and microdots (100 nm in diameter) with a thickness of $50 \mathrm{~nm}$ have been deposited onto $\mathrm{Si}$ wafers. Indexing of Fe continuous thin film's on Si and microdots on GaAs XRD scan confirmed the presence of $\alpha$-Fe BCC phase (ICSD 44863; space group I m3m)[21]. (Figure 1)
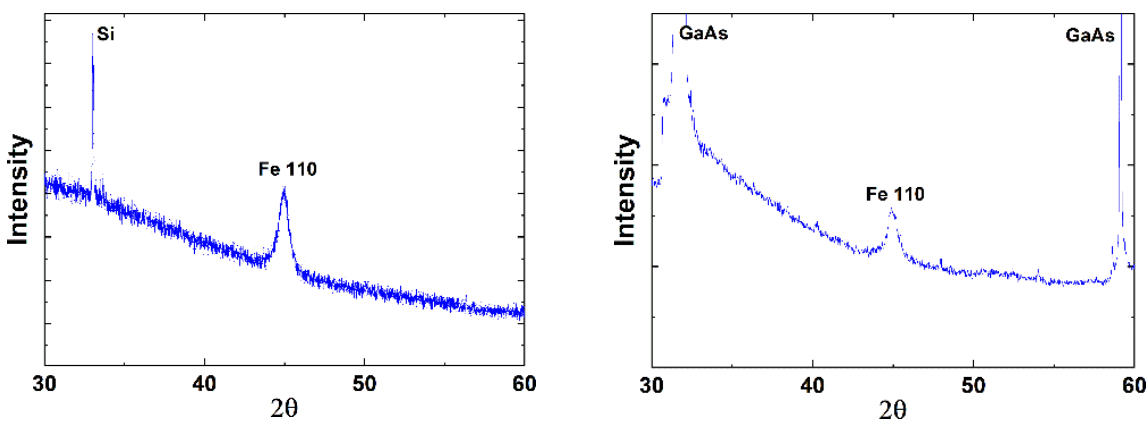

Fig. 1 XRD data of Fe continuous thin film on Si (left) and microdots GaAs (right).

Room temperature hysteresis loops (in-plane) of the Fe continuous films and microdots revealed a soft magnetic behaviour (as expected [22]). (Figure 2) Loops have a square shape, corresponding to the easy axis of the film. Minor room temperature coercivities of $85 \mathrm{Oe}$ and $65 \mathrm{Oe}$ were also observed for Fe continuous films and microdots, respectively. Large $\mathrm{Mr} / \mathrm{Ms}$ ratio of 0.9 and 0.8 has been observed for Fe continuous films and microdots, respectively, which is quite important for high-density recording media applications. [23]
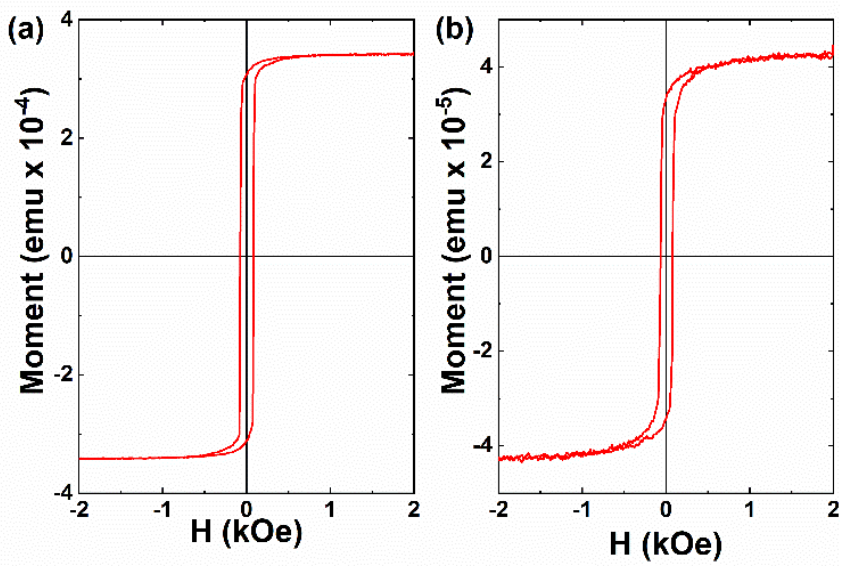

Fig. 2 Room temperature hysteresis loops (in-plane) of Fe (a) continuous film, (b) microdots deposited on Si wafer.

After confirming the data for the continuous and micro-dot Fe films on Si wafers, Fe microdots were synthesized on GaAs substrates. To reduce the mismatch between Fe and GaAs films and increase the quality of epitaxy, the thickness of the micro-dots was limited to $5 \mathrm{~nm}$. [1] Scanning electron microscopy (SEM) image of the Fe micro-dots shows that the structures were successfully synthesized. (Figure 3) 




Fig. 3 SEM image of Fe micro-dots.

As it was stated earlier, the main focus of this work was toward seeing the effect of in-plane anisotropy in micro-dots. Ergo, both in-plane and out-of-plane hysteresis loops were taken at room temperature. While the loop taken in-plane has the characteristic of twophase/magnetic anisotropies behaviour with soft and hard ferromagnetic contributions ( $\mathrm{RT} \mathrm{H}_{\mathrm{C}}=300 \mathrm{Oe}$ ), the out-of-plane loop corresponds to the classical hard direction loop with a room temperature coercivity of 925 Oe. (Figure 4) The reason behind the shape of the inplane loop is the interplay between the two types of magnetic anisotropies; magnetoelastic and magneto-crystalline interface anisotropy. Whilst the latter is of interfacial origin thus due to the bonding of the Fe atoms with As atoms and reconfiguration according to the uniaxial symmetry of the substrate surface (surface anisotropy, [24-26]), the former is due to the anisotropic strain relaxation throughout the volume (anisotropic in-plane strain) of the thin film starting from compressive stress on the interface due to mismatch and later transforms to tensile due to the interfacial phase formation (Fe-Ga-As). These two different anisotropy effects form the basis of the term uniaxial in-plane magnetic anisotropy, ergo the reason behind the behaviour observed in the in-plane hysteresis loop of the $\mathrm{Fe}$ microdots deposited on GaAs substrate. [2]

On contrary, the out-of-plane loop is due solely to cubic anisotropy. It should be noted that thin-film shape anisotropy forces the magnetization to lie in-plane thus out-of-plane direction is hard. [2] It should also be noted that $\mathrm{M}_{\mathrm{R}} / \mathrm{M}_{\mathrm{S}}$ ratio lower than 0.5 on in-plane and out-of-plane loops could be an indication for uniaxial anisotropy contribution produced by internal strains. $[27,28]$ 


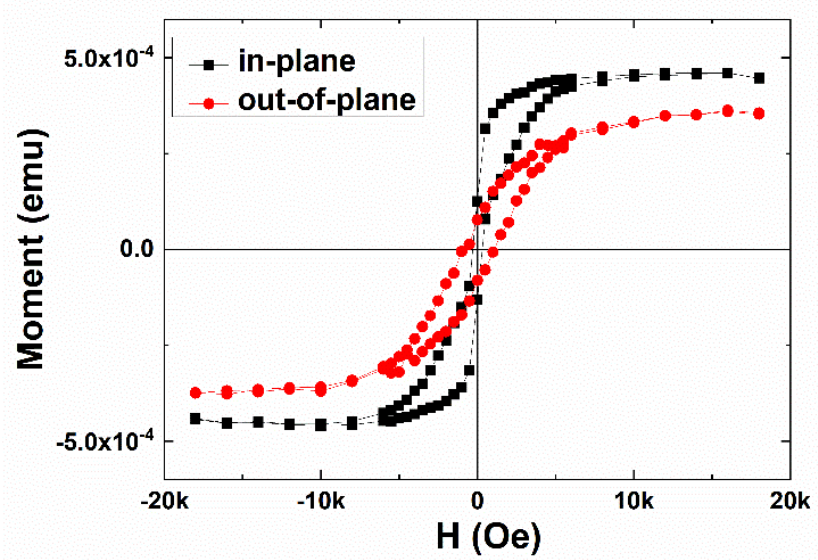

Fig. 4 Room temperature hysteresis loops of Fe microdots deposited on GaAs substrate.

\section{Conclusions}

Fe/GaAs hybrid micro-structures were successfully synthesized by a combinatorial approach of lithography and e-beam deposition techniques. $50 \mathrm{~nm}$ and $5 \mathrm{~nm}$ of Fe was deposited onto Si (001) and GaAs (100) substrates, respectively. Continuous films and microdots were covered with $5 \mathrm{~nm} C r$ to prevent oxidation. XRD scan confirmed the presence of $\alpha$-Fe BCC phase (ICSD 44863; space group I m3m) on Fe continuous films and microdots deposited on Si and GaAs substrates, respectively. SEM image showed that the microdots $(100 \mu \mathrm{m}$ diameter $)$ were decorated throughout the wafer $(100 \mu \mathrm{m}$ in between the microdot disks). Large $\mathrm{Mr} / \mathrm{Ms}$ ratio of 0.9 and 0.8 has been observed for Fe continuous films and microdots deposited on Si substrate, respectively, which is quite important for high-density recording media applications. Moreover, minor room temperature coercivities of 85 Oe and 65 Oe were also observed for Fe continuous films and microdots deposited on Si substrate, respectively. While the loop taken in-plane has the characteristic of two-phase/magnetic anisotropies behaviour with soft and hard ferromagnetic contributions (room temperature $\mathrm{Hc}=300 \mathrm{Oe}$ ), the out-of-plane loop corresponds to the classical hard direction loop with a room temperature coercivity of 925 Oe. In-plane uniaxial magnetic anisotropy was observed similar to the a priori observed effects on continuous films. The underlying reason for the in-plane uniaxial magnetic anisotropy is postulated to be interfacial origin due to the interfacial mixing of Fe atoms and out-diffused GaAs substrate atoms (mainly As). In addition to the interfacial effects, anisotropic strain distribution causes a uniaxial magnetoelastic anisotropy in-plane. Furthermore, MR/MS ratio lower than 0.5 on in-plane and out-of-plane loops could be an indication for uniaxial anisotropy contribution produced by internal strains. Fabricated semi epitaxial Fe microdots could be used in magnetoelectronic devices and micro electro mechanic systems by further improving the interface quality, thus increasing the spin incision efficiency.

\section{Acknowledgement}

Author would like to thank Ozan Akdogan for his helpful discussions. This work was supported by TUBITAK project: 217M322. 


\section{References}

[1] Waldrop JR, Grant RW. Interface chemistry of metal-GaAs Schottky-barrier contacts. Appl Phys Lett. 1979;34(10):630-2. https://doi.org/10.1063/1.90642

[2] Wastlbauer G, Bland JAC. Structural and magnetic properties of ultrathin epitaxial Fe films on GaAs(001) and related semiconductor substrates. Adv Phys. 2005;54(2):137 219. https://doi.org/10.1080/00018730500112000

[3] Moodera JS, Kinder LR, Wong TM, Meservey R. Large magnetoresistance at room temperature in ferromagnetic thin film tunnel junctions. Phys Rev Lett. 1995; https://doi.org/10.1103/PhysRevLett.74.3273

[4] VAN DER MERWE JH. Epitaxial Interfaces. Treatise Mater Sci Technol. 1973 Jan;2:1103. https://doi.org/10.1016/B978-0-12-341802-9.50007-8

[5] Hanbicki AT, van 't Erve OMJ, Magno R, Kioseoglou G, Li CH, Jonker BT, et al. Analysis of the transport process providing spin injection through an Fe/AlGaAs Schottky barrier. Appl Phys Lett. 2003 Jun;82(23):4092-4. https://doi.org/10.1063/1.1580631

[6] Taniyama T, Wastlbauer G, Ionescu A, Tselepi M, Bland C. Spin-selective transport through Fe/AlOx/GaAs(100) interfaces under optical spin orientation. Phys Rev B Condens Matter Mater Phys. 2003; https://doi.org/10.1103/PhysRevB.68.134430

[7] Zega T, Yasar M, Zega T, Yasar M. Interface Effects on Spin Injection in Fe/AlGaAs spinLEDs. APS. 2005;V10.008. https://doi.org/10.1017/S1431927604882850

[8] Hirohata A, Steinmueller SJ, Cho WS, Xu YB, Guertler CM, Wastlbauer G, et al. Ballistic spin filtering across ferromagnet/semiconductor interfaces at room temperature. Phys Rev B. 2002 Jul;66(3):035330. https://doi.org/10.1103/PhysRevB.66.035330

[9] Chye Y, Huard V, White ME, Petroff PM. Properties of a Fe/GaAs(001) hybrid structure grown by molecular-beam epitaxy. Appl Phys Lett. 2002 Jan;80(3):449-51. https://doi.org/10.1063/1.1434302

[10] Klaua M, Kirschner J, Urban R, Myrtle K. Magnetoresistance and magnetic properties of $\mathrm{fe} / \mathrm{cu} / \mathrm{fe} / \mathrm{gaas}(100)$. Phys Rev B - Condens Matter Mater Phys. 1999 Oct;60(14):10242-51. https://doi.org/10.1103/PhysRevB.60.10242

[11] Hirose S, Haneda S, Yamaura M, Hara K, Munekata H. Electronic structure, growth, and structural and magnetic properties of magnetic semiconductor $\mathrm{Fe} / \mathrm{GaAs}$ heterostructures. J Vac Sci Technol B Microelectron Nanom Struct. 2000 Jun;18(3):1397. https://doi.org/10.1116/1.591392

[12] Thibado P, Kneedler E, Jonker B, Bennett B, Shanabrook B, Whitman L. Nucleation and growth of Fe on GaAs(001)- $(2 \times 4)$ studied by scanning tunneling microscopy. Phys Rev B - Condens Matter Mater Phys. 1996;53(16):R10481-4. https://doi.org/10.1103/PhysRevB.53.R10481

[13] Chambers SA, Xu F, Chen HW, Vitomirov IM, Anderson SB, Weaver JH. Simultaneous epitaxy and substrate out-diffusion at a metal-semiconductor interface: $\mathrm{Fe} / \mathrm{GaAs}(001)$ $c(8 \times 2)$. Phys Rev B. 1986 Nov;34(10):6605-11. https://doi.org/10.1103/PhysRevB.34.6605

[14] Mirbt S, Sanyal B, Isheden C, Johansson B. First-principles calculations of Fe on GaAs(100). Phys Rev B - Condens Matter Mater Phys. 2003; https://doi.org/10.1103/PhysRevB.67.155421

[15] Schultz BD, Farrell HH, Evans MMR, Lüdge K, Palmstrøm CJ. ErAs interlayers for limiting interfacial reactions in Fe/GaAs(100) heterostructures. J Vac Sci Technol B Microelectron Nanom $\quad$ Struct. $2002 \quad$ Aug;20(4):1600. https://doi.org/10.1116/1.1491994

[16] Sano K, Miyagawa T. Surface segregations during epitaxial growth of fe/au multilayers on gaas(Ool). Jpn J Appl Phys. 1991;30(7 R):1434-41. https://doi.org/10.1143/ل/AP.30.1434 
[17] Filipe A, Schuhl A, Galtier P. Structure and magnetism of the Fe/GaAs interface. Appl Phys Lett. 1997 Jan;70(1):129-31. https://doi.org/10.1063/1.119284

[18] Farrow RFC, Parkin SSP, Speriosu VS. New approaches to epitaxy of transition metals and rare earths: Heteroepitaxy on lattice-matched buffer films on semiconductors (invited). J Appl Phys. 1988; https://doi.org/10.1063/1.342404

[19] Anderson GW, Hanf MC, Norton PR. Growth and magnetic properties of epitaxial Fe(100) on S-passivated GaAs(100). Phys Rev Lett. 1995 Apr;74(14):2764-7. https://doi.org/10.1103/PhysRevLett.74.2764

[20] Filipe A, Schuhl A. Magnetism of Fe thin layers on GaAs (001). J Appl Phys. 1997; https://doi.org/10.1063/1.364821

[21] Bergerhoff G, Brown ID. Inorganic Crystal Structure Database. Acta Crystallogr Sect A Found Crystallogr. 1981; https://doi.org/10.1107/S0108767381089411

[22] Cullity BD, Graham CD. Introduction to Magnetic Materials. Introduction to Magnetic Materials. Hoboken, NJ, USA: John Wiley \& Sons, Inc.; 2008. https://doi.org/10.1002/9780470386323

[23] Weller D, Doerner MF. Extremely high-density longitudinal magnetic recording media. Annu Rev Mater Sci. 2000 Aug;30(1):611-44 https://doi.org/10.1146/annurev.matsci.30.1.611

[24] Néel L. Anisotropie magnétique superficielle et surstructures d'orientation. J Phys le Radium. 1954; https://doi.org/10.1051/iphysrad:01954001504022500

[25] Krebs JJ, Jonker BT, Prinz GA. Properties of Fe single-crystal films grown on (100)GaAs by molecular-beam epitaxy. J Appl Phys. 1987; https://doi.org/10.1063/1.337886

[26] Jantz W, Rupp G, Smith RS, Wettling W, Bayreuther G. Investigation of single crystal fe films grown by MBE on GaAs substrates. IEEE Trans Magn. 1983;19(5):1859-61. https://doi.org/10.1109/TMAG.1983.1062649

[27] Bitar Z, El-Said Bakeer D, Awad R. Synthesis, characterization, and magnetic properties of nanosized Zn 0.5 Co 0.5 Er x Fe 2-x 04 prepared by coprecipitation method. Turkish J Phys. 2019;43(1):80-92. https://doi.org/10.3906/fiz-1808-22

[28] Ammar S, Helfen A, Jouini N, Fièvet F, Rosenman I, Villain F, et al. Magnetic properties of ultrafine cobalt ferrite particles synthesized by hydrolysis in a polyol medium. J Mater Chem. 2001 Jan;11(1):186-92. https://doi.org/10.1039/b003193n 